hier die Nachbehandlung bedeutend abgekürzt und den Kranken selbst überlassen werden. Allerdings bin ich noch ausser Stande, Anbaltspunkte zu geben, wie lange ejgentlich die Nachbehandlung fortgesetzt werden muss, nachdem alle Erscheinungen der Krankheit beseitigt worden sind. Es müssen das eben weitere und aut einen längeren Zeitraum ausgedehnte Untersuchungen entscheiden. Das Resultat solcher Untersuchungen, wie auch die casuistiscben Belege, gedenke ich in einer künftigen Arbeit zu veröffentlichen, nachdem mir ein hinreichendes Material zu Gebote stehen und eine längere Zeit verflossen sein wird, um vor Ausbleiben von Recidiven gesichert zu sein. Die vorliegende Mittheilung beabsichtigt nur, Anderen das mühsame Experimentiren zu ersparen.

\title{
XII.
}

\section{Wie wirkt das Firnissen der Haut bei Menschen? ')}

Von Prof. Dr. H. Senator in Berlin.

Die Vorstellung von schädlichen Folgen, welche die Unterdrückung der Hautthätigkeit nach sich ziehe, ist zwar, wie es scheint, beinahe so alt, wie die Medicin, hat aber im Laufe der Zeit trotz der fortgeschrittenen Kenntnisse von dem Bau und den Verrichtungen der Haut an Klarheit wenig oder gar Nichts gewonnen. Die alte, dogmatische Medicin hielt einfach an dem Lehrsatz fest, der schon bei Galen sich wiederholt ausgesprochen findet, dass die Verstopfung der unsichtbaren Poren an der Körperoberfläche und die daraus entstehende Zurückhaltung von Dünsten, die "Adiapneustie“, eine Verderbniss der Säfte, Zersetzung u.dgl. zur Folge habe. Sanctorius, welcher nacbwies, dass der Körper in der That durch die Ausdünstung (von Haut und Lungen) wägbare Verluste beständig, wenn auch in wechselnder Menge, erleide, verlieh diesem Lehrsatz eine gewichtige Stütze, Er selbst sprach sich gerade auf Grund seiner

1) Unter "Firnissen“ verstehe ich bier der allgemein üblichen Ausdrucksweise gemäss nicht blos das Ueberziehen der Haut mit einem eigentlichen (0el-) Firniss oder Lack; sondern überhaupt mit fettigen oder klebrigen, der Haut fest anhaftenden Stoffen. 
Körperwägungen dahin aus, dass durch Zurückhaltung der dunstförmigen Ausscheidungen verschiedene Krankheiten, scharfe Säfte ("Acrimonia perspirabilis retenti") entständen, ja er wollte sogar das Verhalten des sogenannten insensiblen Gewichtsverlustes, seine Zuoder Abnahme, zu diagnostischen Schlüssen, allerdings nur von ganz allgemeiner Natur, verwerthen ${ }^{\mathbf{1}}$ ).

Indessen dieser scheinbar so fest begründeten Lehre wurde der Boden wieder entzogen, als man durch die chemische Untersuchung über die Natur der von der Haut ausgedünsteten Stoffe aufgeklärt wurde. Den Hauptbestandtheil derselben bildet bekanntlich der Wasserdampf, neben welchem die geringen Mengen von Kohlensäure und die noch geringeren, gar nicht mehr wägbaren Spuren flüchtiger, riechender Stoffe nicht in Betracht kommen. Seitdem diese Erkenntniss gewonnen wurde, ist wohl ab und zu noch der Versuch gemacht worden, irgend ein unwägbares Ausscheidungsproduct der Haut zu entdecken, auf dessen Zurïckbaltung die Folgen der unterdrückten Hautthätigkeit zu schieben wären, allein bis jetzt ist noch jeder derartige Versuch gescheitert. Es ist allem Anschein nach kein schädlicher Stoff in dem Hautdunst vor-

1) Einige bieranf bezügliche Stellen in seiner: „Ars de statica medica aphorismorum sectionibus VII comprehensa" Lugduni Batavorum 1728. "De ponderatione insensibilis perspirationis sectio prima" lauten:

Aphorism. VIl. "Quantitas perspirationis insensibilis aliquam varietatem patitur, pro varietate naturae, regionis, temporis, aetatis, morborum, ciborum et aliarum rerum non naturalium. "

Aplror. XI. „Si ex staticis deprehendatur, impeditam esse perspirationem, diebus sequentibus, vel succedet plenior perspiratio, vel aliqua evacuatio sensibilis plepior, vel cachexiae vestigium, vel febris."

Aphor. XVI. "Malae qualitates introducuntur, dum corpus uno die est unius ponderis, altero alterius."

Aphor. XL. „Natura, dum in perspirandi officio est impedita, incipit statim in multis deficere."

Aphor. XLlI. "Prima morborum semina tutius cognoscuntur ex alteratione insolitae perspirationis, quam ex laesis officiis."

Aphor. XLIII. „Si ex ponderatione videris, consuetum perspirabile retineri, .... inde cognosces, retentum praenuntiare futuram putredinem.

Aphor. CXIX. „Constipata perspiratione in cervice stupescit sensus pericranii." ....

Aphor. CXXI. „Refrigerationes in morbis acntis indicant mortem, ut in Hermocrate: adimunt enim perspirationem" etc. 
banden, der nicht mit Leichtigkeit auf anderen Wegen, wenn ihm der Weg durch die Haut versperrt ist, aus dem Körper geschafft werden könnte und deshalb kann überhaupt, von einer Zurückhaltung der Perspirationsstoffe, so lange nur die Haut und nicht noch andere Ausscheidungsorgane in ihrer Tbätigkeit gehindert sind, nicht wohl die Rede sein. Mit Rücksicht hierauf sind ja auch in der neuesten Zeit verschiedene andere Erklärungen für die Folgen der unterdrückten Hauthätigkeit aufgestellt worden, doch hat auch von diesen bisher keine sich eine allgemeine Anerkennung verschaffen können.

Aber wenn man auch eine befriedigende Erklärung noch nicht gefunden hat, die Thatsache, dass die Unterdrückung der Hautthätigkeit von schädlichem Einfluss für den Körper ist, gilt darum doch für ganz sicher. Und ganz mit Recht, soweit es sich um Thiere bandelt. Denn bei diesen führt in der That, wie Fourcault und seine zahlireichen Nachfolger gezeigt haben, die Störung der Hautthätigkeit, wie sie beim Ueberfirnissen stattindet, schwere Schädigungen des Organismus und den Tod herbei.

Bei Menschen sind derartige Versuche in der ausgesprochenen Absicht, die Function der Haut zu prüfen, meines Wissens, nicht angestellt worden, abgesehen von einigen Beobachtungen, die ich selbst bereits vor mehreren Jahren gemacht und mitgetheilt habe ${ }^{1}$ ) und auf welche ich noch zurückkommen werde. Vielmehr hat man, wie in so vielen anderen Fragen der Medicin, die an Thieren gemachten Erfahrungen ohne Weiteres auf den Menschen übertragen und sie zu den weitgehendsten Schlüssen verwerthet. Es ist hinlänglich bekannt, dass man auf Grund jener Thierversuche das zahllose Heer dep sogenannten Erkältungskrankheiten hat erk]ären wollen, dass man die verschiedensten Störungen der Gesundbeit, welche im Verlauf oder im Gefolge irgend einer Hautaffection, eines acuten Exanthems, einer Verbrennung oder Erfrierung der Haut, ja selbst starker Hautreize auftreten, für analog denjenigen Störungen, welche das Firnissen bei Thieren hervorruft, betrachtet hat. So fest begründet ist, zumal bei den Pathologen, die Ansicht von der Gefährlicbkeit des Firnissens der Haut auch beim Menschen,

1) S. Untersuchungen über den fieberhaften Prozess und seine Behandlung, Berlin 1873. S. $196-203$. 
dass sie trotz der zahlreichen Erfahrungen, welche gerade auf pathologischem und therapeutischem Gebiete in Bezug auf Undurchgängigkeit der Haut, man kann wohl sagen, täglich gemacht werden und welche alles Andere eher, als eine Gefährlichkeit derselben nabe legen, nicht hat erschüttert werden können.

Wer wüsste nicht, dass zu allen Zeiten die menschliche Haut aus hygienischen, therapeutischen oder kosmetischen Rücksichten ohne Schaden mit allen möglichen Oelen, Fetten und Salben bestrichen, mit Schmierseifen eingerieben, mit Theer überzogen, mit Pflastern in grösster Ausdehnung beklebt, kurz einer Behandlung unterworfen worden ist, welche bei Thieren sicher den Tod herbeiführt! Hat man ferner nicht Menschen mit ausgedehnten Verbrennungen, durch welche allein doch schon ein Theil der Haut leistungsunfähig gemacht wird, Tage lang hintereinander während vieler Stunden in Oelbäder gehalten und so die noch vorhandene unversehrte Haut zur „Perspiration" unfähig gemacht, während man sie doch zu einer verstärkten Thätigkeit hätte antreiben müssen, wenn die Firnissversuche an Thieren hierfür maassgebend gewesen wären! Und endlich, sieht man nicht, dass Menschen, deren Haut über und über mit Schuppen und Borken dick bedeckt ist (z. B. bei Psoriasis, Ichthyosis, Eczema squamosum) und wie ein undurchdringlicher Panzer, dichter vielleicht noch und undurchdringlicher als jeder Firnissüberzug, den Körper umgiebt, keine jener tiefen Störungen darbieten, unter denen die gefirnissten Thiere so schnell zu Grunde gehen ${ }^{1}$ )!

1) Was man gewöhnlich als Bestätigung für die Ansicht von der Gefährlichkeit des Ueberfirnissens der Haut bei Menschen anzuführen pllegt, beschränkt sich auf die Erzählung von einem Knaben, welcher zur Verherrlichung der Huldigungsfeier eines Papstes als goldener Engel erscheinen sollte und zu dem Zwecke am ganzen Körper vergoldet wurde. Er starb aber in der Nacht darauf vor dem Beginn der Feierlichkeit. Es ist kaum nöthig noch besonders hervorzuheben, dass diese Erzähỉung gar Nichts beweist, da man nicht erfăhrt, woran und unter welchen Erscheinungen der Tod eintrat, ob die zum „Vergolden" angewandten Stoffe nicht gifthaltig waren u. s.w. Gerade der angeblich so schnell nach dem Vergolden eingetretene Tod spricht schon gegen jede Analogie mit dem Tode der Thiere nach dem Firnissen. Alle Angaben stimmen nehmlich darin überein, dass die Thiere um so schneller nach dem Ueberfirnissen sterben, je kleiner sie sind, je grösser demnach ihre Oberfläche im Verhältniss zu ihrem Gewicht ist. Daher sind.z. B. Kaginchen so sehr empfindlich gegen diesen Eingriff, aber sie überleben ihn 
Es fehlt also nicht an gelegentlichen Erfabrungen dafür, dass der Mensch jene Eingriffe auf die Haut anders und besser verträgt, als das Thier und es waren eben diese bisher unbeachtet gebliebenen Erfahrungen, welche mich zuerst den Versuch wagen liessen, die Haut bei Menschen zu firnissen und zwar gerade in der Absicht, das Verhalten der. Menschen mit demjenigen gefirnisster Thiere zu vergleichen. Ganz besonders batte ich dabei die Körpertemperatur im Auge, da bekanntlich ein schnelles Abfallen derselben zu den ersten und hervorstechendsten Wirkungen des Ueberfirnissens gèhört. Von diesem Gesichtspunkt aus war es natürlich, dass ich Menschen mit abnorm erhöhter Körpertemperatur, also im fieberhailen Zustand, zu den Versuchen wählte zugleich in der Hoffnung, vielleicht eine therapeutisch verwerthbare Methode dadurch zu gewinnen. Zum Ueberziehen der Haut gebrauchte ich theils Heft-

doch $\frac{1}{3}-4$ Tage und selbst noch länger, Hunde je nach ihrer Grösse leben noch bis zu mehreren Wochen (bis 34 Tage nach Edenhuizen), Pferde boch länger. Darnach, sollte man meinen, müsste ein Knabe, bei dem das Verbältniss von Gewicht zur Körperoberfläche sich doch wohl mit demjenigen eines mittelgrossen Handes ungefähr vergleichen lässt, wenigstens erst mehrere Tage, wenn nicht gar Wochen lang nach dem "Vergolden" sterben, wenn der Tod wirklich demjenigen der gefirnissten Thiere gleichgesetzt werden sollte. Und doch soll er das Vergolden noch nicht einmal einen Tag äberlebt haben. Denn es ist wohl selbstverständlich, dass man ihn nicht schon Tage lang vor der Feierlichkeit in seiner Vergoldung wird baben umherlaufen lassen.

Als Gegensatz zu dieser, für unsere Frage demnach ganz werthlosen Anecdote führe ich das in America noch bis vor nicht langer Zeit geübte Verfabren des "Federns" an, jene Strafe der Volksjustiz, welche darin bestand, den Schuldigen vollständig mit Theer anzustreichen und mit Federn zu bestreuen, ein Verfahren, durch welches der Betroffene wohl der Schande und dem Gelächter preisgegeben aber keineswegs krank gemacht, oder gar getödtet wurde. Hierüber liegen vollständig glaubwürdige Berichte von Augenzeugen vor, unter anderen erinnere ich mich, einen solchen vor mebreren Jabren in der "Gartenlaube" gefunden zu haben. Freilich wird ein solcher „Gefederter" nur ein Paar Stunden höchstens seinen Firnissüberzug behaiten haben, allein man muss andererseits bedenken, dass dabei die Hant in ihrer ganzen Ausdebnung bestrichen wird, was bei allen Thieren sofort eine rapide Temperaturabnahme mit vollständiger Prostration howirkt (Valentín sah die Temperatur bei Kaninchen in wenigen Stunden um $20^{\circ} \mathrm{C}$. fallen!) uad sich doch wohl auch bei jenen Menschen musste bemerklich machen, wenn das Firnissen einen äbnliehen Einfluss hätte. 
pflaster, mit welchem die Haut in verschiedener Ausdehnung dicht beklebt wurde, theils dicke, zähe Salben, theils Collodium, welchem, um es weniger spröde zu machen, etwas Ricinusöl zugesetzt war, theils endlich eine Auflösung von Guttapercha in Chloroform (Traumaticin).

Anfangs bestrich ich in der Meinung, die allgemeine Furcht vor dem Firnissen der Menschen könnte doch am Ende begründet sein, nur höchstens eine Extremität, dann aber, als ich auch nicht die geringste Spur einer Allgemeinwirkung, am allerwenigsten irgend eine der bei gefirnissten Thieren beobachteten Störungen wahrnahm, ubberzog ich grössere Hautflächen, nehmlich ausser einer Extremität noch Brust und Bauch oder Rücken mit einer sogenannten ,imperspirabelen" Decke bald nur einmal, bald öfters hintereinander.

Ueber 5 solcher Beobachtungen, in denen bei fiebernden Menschen grössere Hautstrecken wiederholt gefirnisst wurden, habe ich, wie gesagt, schon früher Mittheilung gemacht. Sie betrafen Typhuskranke. Der Typhus nahm dabei keinen ungewöhnlichen Verlauf, ein Patient, welcher zugleich eine chronische Brustfell- und Lungenentzündung hatte, starb. In Betreff der uns hier beschäftigenden Frage habe ich damals als das Ergebniss meiner Beobachtungen Folgendes gesagt: „dass das Ueberziehen der Haut mit mehr oder weniger imperspirablen Stoffen selbst bis über die Hälfte der Oberfläche und jedenfalls in einer Ausdehnung, welche bei Thieren entschieden schädliche Folgen hat, bei Menschen ohne Nachtheil bleibt, geht aus diesen wenigen Beobachtungen schon mit Sicherheit hervor; ebenso ist mehrmals ein Einfluss auf die Temperatur unverkennbar, wenn auch im Allgemeinen der Erfolg ein sehr ungleicher war und die Temperaturerniedrigung, wenn überhaupt, bald schneller, bald langsamer nach dem Ueberfirnissen eintrat".

Da diese Firnissversuche nur bei kranken Menscben und auch nur bei einer einzigen Krankheitsform, dem Abdominaltyphus angestellt worden sind, so kann man mit Recht fragen, ob sie denn zu allgemeinen Schlüssen in Bezug auf den Menschen überhaupt, zumal den gesunden Menschen, berechtigeu. Es wäre ja nicht unmöglich, dass bei den tiefen Stoffwechselveränderungen, welche in fieberhaften Krankheiten und beim Typhus insbesondere, stattinden, der Ausfall der Hautthätigkeit oder wenigstens deren theilweise Beschränkung gar nicht füblbar würde. Ja, da die Haut von Fiebernden 
doch meistentheils heisser und ihre Gefässe wenigstens stellenweise oder zu gewissen Zeiten weiter als normal sind, so liegt es gar nicht einmal sehr fern, sich vorzustellen, dass gerade im Fieber, wenn ein Theil der Haut leistungsunfähig gemacht wird, der übrigbleibende Theil mit Leichtigkeit, leichter sogar, als in nicht fieberbaften Zustande den Ersatz übernehmen könne.

Ein anderes Bedenken gegen die Zulässigkeil eines allgemeinen Schlusses aus jenen Versuchen, welches ich mir.schon damals nicht verbeblt habe, ist dies, dass die Ausdehnung der gefirnissten Stellen selbst da, wo grössere Strecken der Hautoberfläche gleichzeitig bedeckt waren, noch nicht hingereicht habe, um bei erwachsenen Menschen Störungen hervorzurufen, oder dass der Ueberzug nicht lange genug der Haut angehaftet habe. Denn bei Thieren hängt, wie ich schon angeführt habe, der Grad der Störungen : und die Schnelligkeit, mit weleher sie eintreten, hauptsächlich von der Grösse ab, so dass man bei grösseren Thieren weit mehr von der Haut und längere Zeit hindurch firnissen muss, bis sie erkranken, als bei kleineren.

Aus diesen Gründen habe ich weitere Beobachtungen und Versuche angestellt, von denen ich drei ausführlicher hier mitheile, gegen welche keines der genannten Bedenken noch, wie ich meine, irgend ein anderes wird erhoben werden können. Namentlich, was die Ausdehnung und die Dauer der Firnissung sowie die Beschaffenheit des Hautüberzuges betrifft, können diese Beobachtungen;, wie mir scheint, denjenigen Firnissungen, welche sich bei Thieren als die allergefährlichsten erwiesen baben, dreist an die Seite gesetzt werden.

Man kann bei Menschen, die man doch in ihren Bewegungen nicht ganz und gar beschränken und denen man nicht unnöthige Schmerzen verursachen will, nicht alle jene Stoffe zum Firnissen wählen, die bei Thieren zu diesem Zwecke angewandt worden sind. Auch ist man bei Menschen durch mancherlei äussere Rücksichten in der Auswabl der Stoffe mehr beschränkt, als bei Thieren, nicht am wenigsten durch die Rücksicht darauf, dass derjenige, an welchem solche Versuche gemacht werden sollen, für die er gar kein wissenschaflliches Interesse hat, nicht Zweifel fasse an der Nothwendigkeit, Nützlichkeit oder mindestens an der Unschädlichkeit des Verfahrens.

Aụs meinen früheren und manchen späteren Erfahrungen nun 
habe ich es am besten allen Anforderungen entsprechend gefunden, wenn die Extremitäten mit gut klebendem Heftpflaster dicht eingewickelt, der Rumpf aber mit Collodium ricinatum dick bestrichen wurde. Zur grösseren Sicherheit wurden noch die ersten und die leizten Windungen der Heftpflastereinwickelung, sowie andere Stellen, an welchen etwa eine Entblössung der Haut durch Bewegungen hätte eintreten können, wie z. B. an den Gelenken, ebenfalls noch mit Collodium überpinselt und so noch besonders geschützt. Auch wurde stets auf etwas schadhaft gewordene Stellen des Ueberzuges geachtet, um sie sofort auszubessern.

Eine solche Art von Hautüberzug führt, wenn sie Tage lang bestehen bleibt, bei Thieren sicher den Tod herbei. Von der Heftpflastereinwickelung wird dies wohl Niemand bezweifeln, denn von allen den bei Thieren mit tödtlichem Erfolg angewandten Firnissbekleidungen (Leim, Eiweiss, Theer, Oel, Gummi etc.) können sich die wenigsten oder vielmehr kann sich keine mit jener in Bezug auf Haftbarkeit und Undurchgängigkeit messen. Eher könnte man an der Schädlicbkeit des Collodiumüberzuges zweifeln, zumal da gewöhnlich bei den Thierversuchen von "imperspirabelen" Ueberzügen und von Unterdrückung der "Hautperspiration" geredet wird, während die Collodiumdecke gewiss nicht durchaus undurchgängig für die Hautausdüustung ist ${ }^{1}$ ). Da auch bisher, meines Wissens, mit Collodium an Warmblütern keine Firnissversuche angestellt worden sind $^{2}$ ), so habe ich selbst, um jedem Einwand zu begegnen, einen solchen angestellt, der denn auch zeigte, dass das Firnissen mit Collodium ebenfalis tödtlich wirkt. Da der Versuch auch in anderer Beziehung von Werth ist, weswegen ich noch einmal werde auf ihn verweisen müssen, so theile ich ihn hier mit.

Fin mittelgrosses Albino-Kaninchen von $1200 \mathrm{Grm}$. Gewicht zeigte mehrere Tage (16.-18. November) vor jedem Eingriff bei mehrmals tăgltch vorgenommenen Messungen $5-6 \mathrm{Cm}$. tief im Rectum eine Temperatur zwischen $38,6-39,0^{\circ} \mathrm{C}$. bei einer Zimmertemperatur von $9-13^{0} \mathrm{R}$.

7) Dies gilt auch für manche der bei Thieren benutzten und als „imperspirabel“ bezeichneten Stoffe z. B. für arabisches Gummi.

2) Frösche hat W. Berg (Untersuch. über die Hautathmung des Frosches. Dorpat 1868) mit Collodium hepinselt. Es erwies sich für diese unschädlich, ebenso wie Malerfirniss. 
Am 18. November Mittags wird es am ganzen Rumpf zlemlich kurz geschoren '). Am Abend desselben Tages, etwa 5 Stunden nach dem Scheeren, betrug die immer bei gleicher Tiefe im Rectum gemessene Temperatur $38,8^{\circ} \mathrm{C}$. bei einer Zimmertemperatur von $13^{\circ} \mathrm{R}$. In den folgenden Tagen (bis zum 25. Novbr.) schwankte die Temperatur im Rectum von $38,7-39,2^{0}$ bei einer Zimmertemperatur von $8-11^{\circ} \mathrm{R}$.

Am 26. Novbr. früh 9 Uhr bei einer Zimmertemperatur von $7,5^{0} \mathrm{R}$. misst es im Rectum $39,0^{\circ} \mathrm{C}$. Der aus der Blase gedrückte Urin ist alkalisch, trübe von Erdsalzen, wird klar auf Zusatz von Salpetersäure und bleibt es dann auch beim Kochen. Jetzt werden Rücken und Seiten, nachdem die nachgewachsenen Hare wieder kurz geschoren worden, mit Collod. riciatam bestrichen, 9 Uhr 25 Min. Temp. 37,550 Zimmer $7,5^{\circ} \mathrm{R}$.

\begin{tabular}{|c|c|c|c|c|}
\hline - & -35 & - & $-37,2^{0}$ & \\
\hline 10 & - & - & $37,6^{0}$ & Zimmer $8^{0} \mathrm{R}$. \\
\hline 4 & $-\mathbf{3 0}$ & - & $38,1^{0}$ & - $\quad 8^{\circ} \mathrm{R}$. Urin, wie heute früh. \\
\hline Am 27. Nov. früh 9 & -30 & - & $38,4^{0}$ & $g^{0} \mathrm{R}$. Das Thler ist ganz munter. \\
\hline Mittags 3 & -45 & - & $39,1^{\circ}$ & $8,5^{0} \mathrm{R}$. \\
\hline cden & & 0 & $\begin{array}{l}\text { eiten } \\
\text { a tach }\end{array}$ & $\begin{array}{l}\text { an Nenem und ansserdem d } \\
\text { estrichen. }\end{array}$ \\
\hline
\end{tabular}

4 Uhr 5 Min. Temp. $36,6^{0}$

$7-30-\quad 37,1^{0}$ Zimmer 11,00 R.

Am 28. Nov. früh 8 Uhr $45 \mathrm{Min}$. Temp. 38, $4^{0}$ Zimmer $9^{0} \mathrm{R}$. Uirin klar, sauer, trübt sich beim Kochen (Erdsalze), blärt sich aber auf Zusatz von Salpetersäure.

Abends 5 Uhr 30 Min. Temp. $38,2^{\circ}$ Zimmer $11^{\circ} \mathrm{R}$.

Der Ueherzug wird überall erneuert.

Am 29. Nov. früh 9 Ubr - Min. Temp. 37,7 $7^{\circ}$ Zimmer 10,50. Die Respiration ist sehr verlangsamt. Urin wie gestern, nur bleibt bei Zusatz von Salpetersäure zum gekochten Urin eine geringe Trübung (Eiweiss?) bestehen.

Am 30. Nov. früh 9 Uhr - Min. Temp. 37, $15^{0}$ Zimmer 10,50 R. Resp. 40-42 in der Min. Abends $5=30-\quad-37,3^{0}=13,0^{\circ}$ R. Urin enth.etwas Eiweiss. Am 1. Dec. früh 9 - - - - $35,4^{0} \quad-\quad 10,5^{0} \mathrm{R}$. Das Thier hat Diarrhoe. Abends 5 - 30 - $\quad-34,2^{0}$.

Am 2. Dee. früb todt gefunden. Der aus der Blase genommene Urin ist sauer und enthält eine Spur Eiweiss aber keine Formelemente.

Der Ausgang dieses Versuches beweist, dass das Pinseln mit Collodium sich den anderen Firnissungen ebenbürtig anreiht. Und somit glaube ich die von mir bei Menschen gewählte Methode, die Haul zu uberziehen, als ganz gleichwerthig den Firnissungen der Thiere bezeichnen $z u$ dürfen.

1) Es ganz kahi zu scheeren oder zu rasiren habe ich vermieden, um das Brennen auf der Haut, welches das Collodium seines Aethergehaltes wegen auf der zarten Kaninchenhaut hätte hervorrufen können, za vermeiden. 
Nur in den beiden ersten der hier folgenden Beobachtungen ist diese Art des Hautuberzuges mit Heftpflaster und Collodium zur Anwendung gekommen. Er wurde übrigens in so grosser Ausdehnung, als es die Umstände nur irgend zuliessen, gemacht und Tage lang in gleicher Ausdehnung und Vollständigkeit erhalten. In der dritten Beobachtung wurde das Firnissen mit Theer ausgefuhrt. Sie übertrifft die ersteren beiden noch in Bezug auf Ausdehnung der gefirnissten Oberfläche und was den zum Firnissen gewählten Stoff, den Theer, betrifft, so ist er wiederholt bei Thieren angewandt worden - mit demselben Erfolg, wie alle anderen sogenannten Firnisse.

In allen 3 Fällen wurde eine besondere Beachtung dem Verhalten der Temperatur und des Harns geschenkt. Jene wurde Morgens und Abends in der Achselhöble gemessen, der Harn mit besonderer Sorgfalt auf Eiweiss untersucht und zwar jedesmal durch Kochen und Zusatz von Salpetersäure, sowie durch Essigsäure und concentrirte Lösung von schwefelsaurer Magnesia, zuweilen auch ausserdem noch durch Essigsäure und Ferrocyankalium. Ich lasse aus den Beobachtungs-Journalen alles Dasjenige fort, was für die Beurtheilung der in Rede stehenden Frage ohne Belang ist ${ }^{1}$ ).

I. Wilhèlm L., Particulier, 40 Jahre alt, wurde am 22. September 1875 mit einer subacuten rbeumatischen Polyarthritis auf die innere Station des AugustaHospitals aufgenommen. Er ist ejn kräftig gebauter Mensch von mässig gutem Ernährungszustande und glebt an, schon im Jabre 1873 an derselben Krankheit und zwar damals $3 \frac{1}{2}$ Monate lang gelitten zu haben. Seine gegenwärtige Krank. heit besteht seit 14 Tagen. Sie erwies sich im Verlauf als sehr hartnäckig. Er hatte schon ausserhalb des Krankenhanses ärztliche Hülfe gehabt und wurde von uns mit Carbolsäure- und Morphium-Einspritzungen, mit Ableitungen, hydropathischen Umschlägen und Bädern bebandelt, doch war der Erfolg sehr wechselnd. Innerlich nahm er Kali nitr., dann Jodkalium, Aconit etc. Das anfänglich bestehende mässige Fieber verlor sich gegen die Mitte des Monats November gänzlich. In dieser Zeit wurde versucht, um ihn von dem Gebrauch des Morphiums zu entwöhnen und da er Chloralhydrat schlecht vertrug, den durch Schmerzen sehr gestörten Scblaf durch Milchsäure zu erzielen. Er nahm davon vom 14.-21. November fast tăglich mebrere Gramme, indess mit nur geringem Nutzen, vielleicht weil die Gabe zu klein war; doch wurde von grösseren Gaben abgesehen, weil er nach

1) Herro Dr. H. Wegscheider, damaligem Assistenten der inneren Station, welcher in den beiden ersten Fällen die Einwickelnngen und Einpinselungen überwachte und zum Theil selbst besorgte, bin ich bierfür $z u$ besonderem Dank verpflichtet. 
der Sănre Appetitlosigkeit, Tebelkeit u. dgl. m. hekam. Man kehrte zur ahendlichen Verabrejchung von Morphium (theils innerlich mit Aq. amygdal. amar., theils subcutan) zurück. Im Januar und namentlich zu der Zeit, als die Firnissversuche gemacht wurden, nahm er ausserdem als Arznei Jodkalium abwechselnd mit Colchicum und Aconit. Der Pat. Jag während der ganzen Versuchszeit mit Hemde und leichter Jacke bekleidet im Bett, mit einer oder zwei wollenen Decken bedeckt, vor und nach dieser Zeit brachte er eine Stunde selten länger ausserhalb des Bettes zu. Die Temperatur der umgebenden (Baracken-) Luft war ziemlich scbwankend, wie dies in den Baracken des Augusta-Hospitals während des Winters nicht selten ist. Sie überstieg niemals $15^{0} \mathrm{R}$., fiel aber sehr häufig, namentlich in den früben Morgenstunden, beträchtlich darunter.

Am 26. December warde mit den Versucben begonnen und zwar durch Einwickelung des rechten Unterschenkels. Während der Versuchszeit erbielt Pat. ausser der innerlichen Abenddosis zwei Mal eine Einspritzung von Morphium (15 Milligr.) in die Gegend des linken Fussgelenks, welches sich am meisten durch Schmerzhaftigheit auszeichnete, und zwar ein Mal am 11. Abenis, zu welchem Zwecke einige Streifen Heftpllasters für die Dauer der Einspritzang entfernt wurden, worauf die Einwickelung wieder vollständig hergestellt wurde, dann am 18. Abends, als das Glied bereits wieder von der Einwickelnng befreit war. Das Weitere besagt die folgende Zusammenstellung.

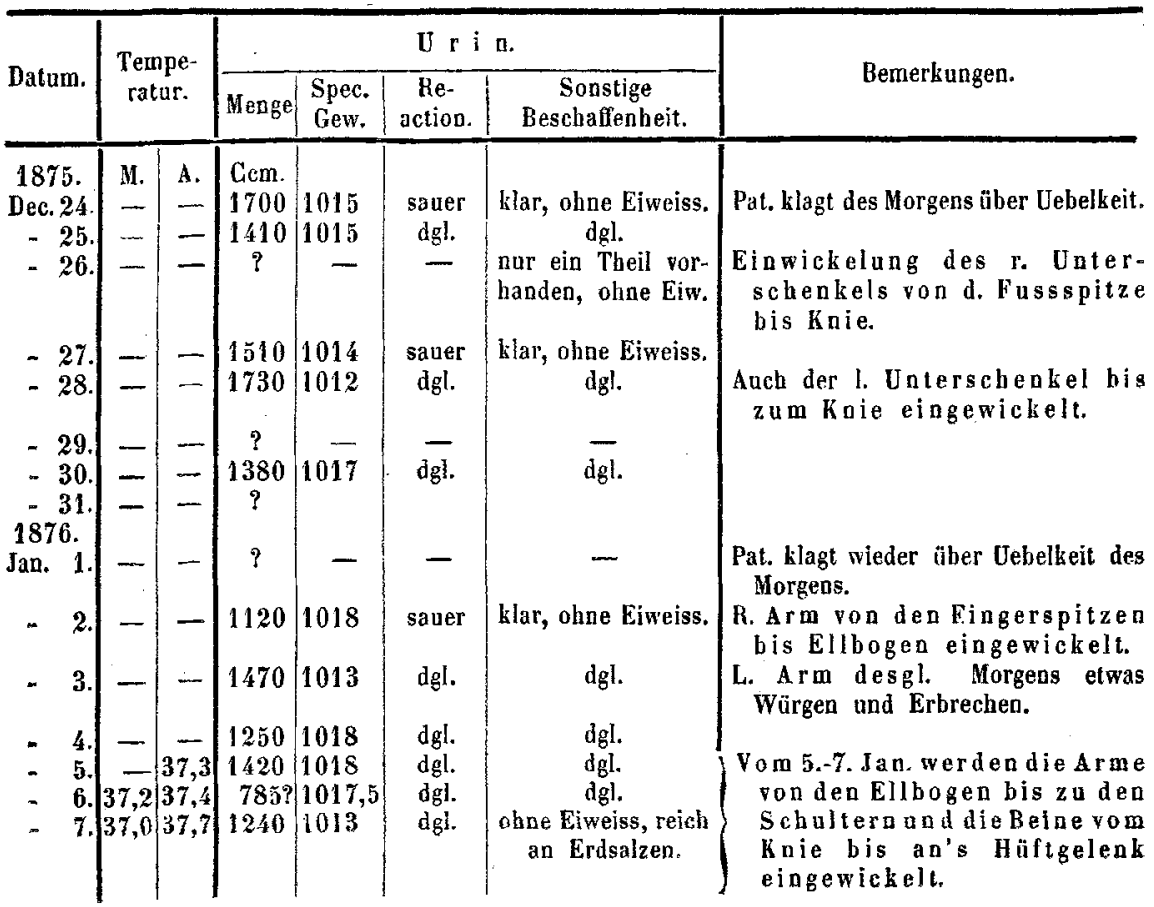




\begin{tabular}{|c|c|c|c|c|c|c|c|}
\hline \multirow{2}{*}{ Datum. } & \multirow{2}{*}{\multicolumn{2}{|c|}{$\begin{array}{l}\text { Tempe- } \\
\text { ratur. }\end{array}$}} & \multicolumn{4}{|c|}{$\mathrm{U} \mathbf{r} \mathrm{i}$. } & \multirow{2}{*}{ Bemerkungen. } \\
\hline & & & Menge & $\begin{array}{l}\text { Spec. } \\
\text { Gew. }\end{array}$ & $\begin{array}{r}\text { Re: } \\
\text { action. }\end{array}$ & $\begin{array}{c}\text { Sonstige } \\
\text { Beschaffenheit. }\end{array}$ & \\
\hline 1876. & M. & A. & Gem. & & & & \\
\hline Jan. 8. & 36,9 & 37,5 & 1380 & 1012 & sauer & klar, ohne Eiweiss. & \\
\hline & 37,0 & 37,7 & 1130 & 1013 & dgl. & dgl. & $\begin{array}{l}\text { Rücken mit Collod. ric. bestri- } \\
\text { chen und bis am 18. täglich } \\
\text { vou Neuem. }\end{array}$ \\
\hline-10. & 37,2 & 37,5 & 1765 & 1014 & $\begin{array}{c}\text { schwach } \\
\text { sauer }\end{array}$ & $\begin{array}{l}\text { blasser als sonst u. } \\
\text { etwas trübe obne } \\
\text { Eiweiss. }\end{array}$ & $\begin{array}{l}\text { Desgl. Brust and Bauch bis } \\
\text { unterhalbdes Nabels and bis } \\
\text { zum j8. täglich von Neuem. }\end{array}$ \\
\hline-11. & $37, \mathbf{3}$ & 37,4 & 1530 & 1013 & $\mathrm{dgl}$. & $\mathrm{dgl}$. & $\begin{array}{l}2 \text { um } \\
\text { Einspritzung von Morphium (s. oben). }\end{array}$ \\
\hline-12. & 37,2 & 37,5 & 1670 & $|1012|$ & dgl. & dgl. & $\begin{array}{l}\text { Pat. klagt wieder mehr über Uebel- } \\
\text { keit und Kopfschmerzen. }\end{array}$ \\
\hline-13. & 37,3 & 37,4 & 1370 & 1016 & dgl: & dgl. & Keine Uebelkeit. \\
\hline-14. & 37,4 & 37,4 & 1380 & 1014 & dgl. & $\begin{array}{l}\text { blass, trübe; bis zum } \\
18 \text {, enthält d. Filtrat }\end{array}$ & $\begin{array}{l}\text { Pat. klagt über efwas Brennen beim } \\
\text { Urinlassen. }\end{array}$ \\
\hline-15. & 37,2 & 37,3 & 1460 & 1016 & dgl. & d. Harn s ganz geringe & Keine Debelkeit, keine Kopfschmerzen. \\
\hline 16. & 37,4 & 37,5 & 1610 & 1011 & $\mathrm{dgl}$. & Spuren v. Eiweiss. In & Desgl. \\
\hline 17. & 37,2 & 37,8 & 1400 & 1010 & neutral & d. mässigen Sediment & Kein Brennen nehr beim Urinlassen. \\
\hline-18 & 37,5 & 37,6 & 1570 & 1011 & $\begin{array}{l}\text { schwach } \\
\text { sauer }\end{array}$ & $\begin{array}{l}\text { Eiterkörperchen und } \\
\text { Blasenepithelien. }\end{array}$ & $\begin{array}{c}\text { Einwickelungdes l. Ober-und } \\
\text { Unterschenkels entfernt. }\end{array}$ \\
\hline-19 & 37,2 & 37,0 & $1300 ?$ & 1013 & dgl. & klar, olıne Eiweiss. & $\begin{array}{l}\text { Vormittag beide Arme, Nach- } \\
\text { mittag r. Bein befreit. }\end{array}$ \\
\hline-20 & 36,4 & 37,5 & 1310 & 1010 & $\mathrm{dgl}$. & $d g$ & \\
\hline 21. & 37,3 & 37,6 & 1740 & 1019 & dgl. & agk. & \\
\hline 22 & 81 & & 1840 & 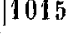 & sauer & $\mathrm{nz} \quad \mathrm{nOI}$ & \\
\hline
\end{tabular}

Wie man siebt, waren vom 10.-18. Januar, genau eine. Woche, beide Beine von den Zehenspitzen bis zu den Hüten, beide Arme von den Fingerspitzen bis zu den Schultern mit Heftpflaster dicht bekleidet und ausserdem der gröste Theil des Rumpfes mit einer dicken Schicht von Collodium bedeckt. Frei waren in dieser Zeit nur Kopf und Hals, Gesäss - und Scbamgegend.

II. Heinrich S., Stellmacher, 36 Jahre alt, wurde am 13. Januar 1876 mit chronischer rheumatischer Affection der Gelenke und Muskeln aufgenommen. Er will schon seit October 1875 krank und seit etwa 3 Wochen (Weihnachten) bettlägerig gewesen sein. Er ist kraftig gebaut, ziemlich mager, Appetit und Stubl sind normal. Er wurde sofort mit Salicylsäure and salicylsaurem Natron mit ziemlich gutem Erfolg behandelt. Es blieben nehmlich, nachdem er sich von den Nachwirkungen der Salicylsüure erholt hatte, pur vage Schmerzen in den Gliedern zurück, während die Gelenke selbst abschwollen. Er wurde nun in gleicher Weise, wie der Mann in der ersten Beobachtung mit Heftpflaster und Collodium bedeckt. Das Nähere ergiebt die Tabelle; 


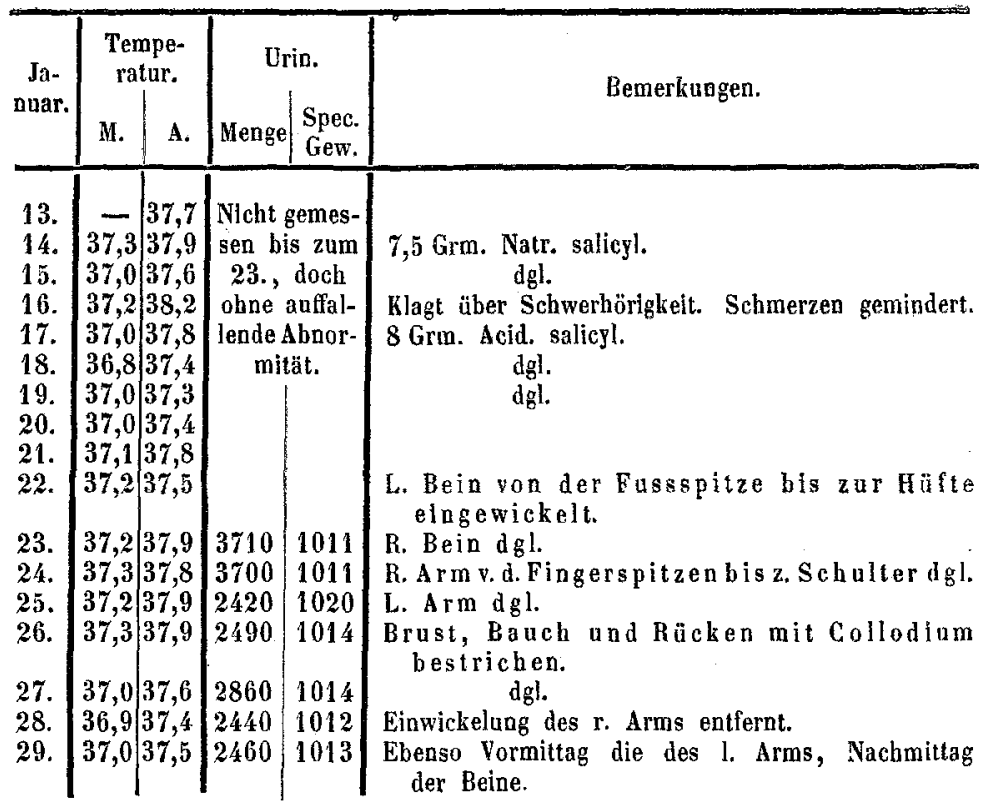

Der Urin war von Anfang bis zu Eade klar, ohne Spur yon Eiweiss. Seine Menge wurde im weiteren Verlauf normal. Am 26. und 27. war die Haut in derselben Ausdehnung wie bei l. vollständig überzogen, 3 Tage lang (25. - 27.) waren nur die vier Extremitäten vollständig bedeckt. Länger konnte die Beobachtung nicht ausgedehnt werden, da die Bedeckung dem Pat., der sich für geheilt bielt, lästig wurde. Er verliess das Krankenhaus Anfangs Februar.

III. Ida W., Vorkosthändlersfrau, 37 Jahre alt, wurde am 25. April $18 \% 6$ auf die chirurgische Abtheilung des Augusta-Hospitals wegen Pemphigus chronicus aufgenommen ${ }^{1}$ ). Der ganze Körper einschliesslich des Kopfes und Gesichts war mit Pemphigusblasen bedeckt. Nachdem Anfangs warme Wasserbäder mit und olne Zusatz von Sublimat in Gebrauch gezogen waren, gegen welche übrigens die Kranke wegen des durch sie verursachten Brennens sich sehr sträubte, wurde am 2. Mai zu Theereinpinselungen äbergegangen. Es wurden mit dem gewöbnlichen käuflichen Theer zuerst die Unterschenkel und zwar vollständig von den Zehen und Fusssohlen an mit einem Pinsel dick bestrichen, dann die Oberschenkel, die Arme, Rücken, Brust und Bauch, Hals und Gesicht. Auf den behaarten Kopf wurde nach dem Scheeren der Haare eine schneller trocknende Mischung aus gleichen Theilen von 01. Rusci und Alkohol angewandt, ausserdem wurde er ofter mit Leberthran

1) Die Notizen äber diesen Fall, den Ich selbst wiederholt gesehen habe, verdanke ich der Freundichkeit des Herrn Dr. Sticker, damaligem Assistenten der chirurg. Abtheilung. 
stark eingeschmiert. Die einmal eingepinselten Stellen wurden bis zum Abbrecheti der Kur täglich von Neuem bestrichen.

In der Zeit rom 12. oder 13. his zum 22. Hai, jedenfalls länger als eine ganze Woche war buchstablich die ganze Körperoberfläche mit Ausnabme einer kam Handteller grossen Stelle an der recbten Hüfte, welche gesund schien, mit Theer bezw. mit 0l. Rusci dicht überzogen. Die Kranke befand sich dabei, etwas Brennen der Haut abgerechnet, gerade so wie vor und nach dieser Zeit. Am 23. Mai wurde sie ein wenig gebessert entlassen und begab sich in die Charité, wo sie sich auf der Abtheilnng des Herrn Prof. Lewin noch jetzt (December 1876) befindet.

Das Verhalten der Temperatur zeigt die nachfolgende Tabelle.

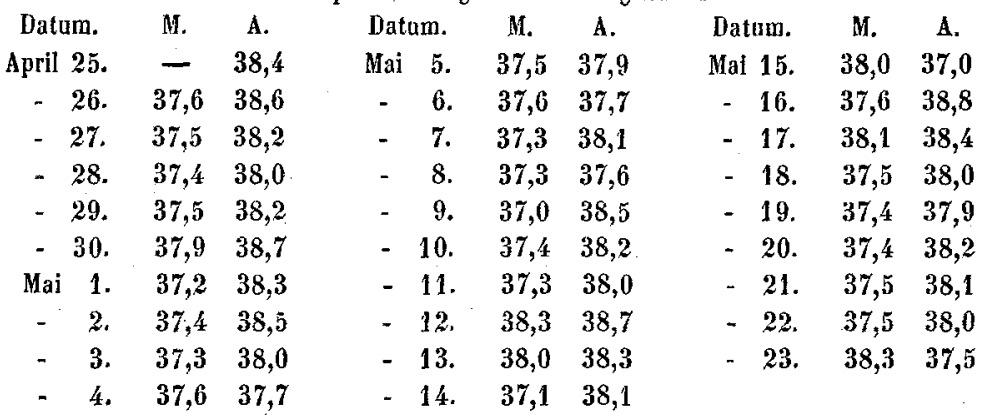

Der Urin, welchen ich während der Zeit wo der Hautüberzug vollständig war, täglich selbst untersucht habe, nahm g]eich nach den ersten Einpinselungen die bekannte dunkle Färbung, an. Auf Zusatz von Schwefelsäure liess er deutlichen Theer- (Phenol-) Geruch erkennen, mit Eisenchlorid förbte er sich violett. Er war fast stets klar, hatte nur zuweilen eine geringe Trübung von einzelnen Plattenepithelien und Schleimkörperchen. Elweiss enthielt er niemals.

Noch mehr Versuche in so grosser Ausdebnung anzustellen, dazu habe ich mich nicht entschliessen mögen, erstens, weil es die Versuchsperson; selbst wenn sie von der dringenden Nothwendigkeit überzeugt ist, keine geringe Ueberwindung kostet, Tage lang einen solchen Pflaster - oder Firnissüberzug auf der ganzen Haut zu ertragen. Dann weil es mir überflüssig erschien bei dem übereinstimmenden Ergebniss, welches auch den früheren Versuchen und vor Allem demjenigen entsprach, was nach den oben angeführten alltäglichen Erfahrungen von vornherein erwartet werden durfte. Dass ich aber ausserdem noch oft genug einen grossen Theil der Körperoberfläche, z. B. beide Beine bis zu den Hüften, oder selbst alle vier Extremitäten mit einem dichten Panzer von Heftpflaster umgeben und Tage lang in demselben erhalten habe mit demselben Erfolg, will ich nicht unerwähnt lassen, da dies 
immerhin zur Bestätigung dessen, was jene drei Hauptversuche ergeben haben, dienen kann. Uebrigens sind gewiss ähnliche Beobachtungen mit weit ausgedebnten wenn auch nicht gerade die ganze Haut bedeckenden Heftpflasterverbänden oder mit Theerfirnissungen auf chirurgischen Abtheilungen oder in Anstalten für Hautkranke oft genug gemacht worden und haben ihre Unschädlichkeit dargethan. Ob dabei auf das Verhalten der Temperatur und des Harns besondere Rücksicht genommen wurde, ist mir nicbt bekannt. Es dürfte nur erwünscht sein, Näheres darüber zu erfahren.

Was nun den Erfolg jener Versuche betrifft, so war er, wenn man das Verhalten gefirnisster Thiere im Auge hat, ein vollständig negativer. Von allen jenen bedrohlichen Erscheinungen, welche selbst grössere Thiere darbieten, wenn auch nur die Hälfte ihrer oberfläche gefirnisst ist, war gar nichts zu bemerken. Nichts von der jähen Temperatursenkung, von dem schnellen Kräfteverfall, nichts von Dyspnoe, von Krämpfen und Lähmungen, keine Albuminurie, keine Diarrhöen, keine hydropischen Ergüsse. Man wird nicht eirwenden dürfen, dass es sich hier um Kranke und nicht um gesunde Menschen gehandelt habe. Allerdings waren die drei Versuchspersonen nicht gesund, allein wenn ich auch gar nicht betonen will, dass kranke Individuen im Allgemeinen ja viel weniger wiederstandsfähig sind als gesunde und deshalb schädliche Folgen irgend eines Eingriffes viel eher und in höherem Maasse zeigen mussten, wenn ich auch ferner keinen Werth darauf legen will, dass die Uebel, mit denen die Versuchspersonen behaftet waren, durchaus nicht zu denjenigen gehören, welche edle und zum Leben nothwendige Organe betreffen, dass sie häufig genug das Leben in keiner Weise verkürzen und mehr örtliche als allgemeine Störungen hervorrufen - so viel beweisen die Beobachtungen jedenfalls, dass das Firnissen der Haut, d. h. dasjenige, was gewöhnlich als "Unterdrückung dèr Hautthätigkeit" bezeichnet wird, bei Menschen kein tödtlicher und auch kein krankmachender Eingriff ist.

Uebrigens war der Pat. Heinrich S. (II.), als der Versuch mit inm gemacht wurde, beinahe Reconvalescent. Wenn aber doch einmal der Umstand, dass Kranke zu den Versuchen dienten, in Berücksichligung gezogen werden soll, so darf wohl bemerkt werden, dass zwei von den Dreien an einer Krankheit (dem sogenannten 
Rheumatismus) litten, für welche die „Unterdrückung der Hautthätigkeil" mit Vorliebe als ursächliches Moment in Anspruch genommen wird, denen also jener Eingriff auf die Haut erst recht verderblich hätte werden müssen. Er ist es gleichwohl nicht geworden ${ }^{1}$ ).

Einige untergeordnete Erscheinungen traten allerdings während der Versuchszeit auf und es wầre noch deren etwaiger Zusammenhang mit der Bedeckung der Haut zu prüfen:

Im Fall I wurde wiederholt Uebelkeit und zwar in den Morgenstunden, einmal auch etwas Erbrechen beobachtet. Icb glaube diese übrigens immer schnell vorübergegangenen Störungen nicht auf das Firnissen schieben zu müssen, da der betreffende Patient, dessen Magen ohnehin durch allerband Arzeneien, namentlich auch durch die Milchsäure (s. oben) angegriffen war, schon vorher öfters über Uebelkeit geklagt hatte, wahrscheinlich in Folge des anhaltenden Morphiumgebrauchs. Von der nach längerer Unterbrechung wieder einmal am 11. gemachten Morphiumeinspritzung ist wohl auch der Tags darauf vorhaudene Kopfschmerz abzuleiten. Ein Zusammenhang dieser geringfügigen Beschwerden mit Firnissung ist um so mehr von der Hand zu weisen, als sie während der Dauer der letzteren wieder schwanden. - Dagegen sind die Veränderungen des Urins, welche gleich nach vollendeter Einwickelung aller 4 Extremitäten eintraten und von einer allerdings nur geringen entzündlichen Reizung der Blase abhingen (Abnahme der sauren Reaction, Beimischung von Blasenepithelien und Eiterkörperchen und von geringen Eiweissmengen) wohl ganz sicher Folgen der Heftpflasterbedeckung und zwar der Resorption von Bestandtheilen des Pflasters, welche leicht eine Reizung der Harnwege ausüben (Terpentin u. a. m.). Das tagelange Verweilen des Pflasters auf der Haut verbunden mit den unvermeidlichen Druck- und Reibebewegungen haben die Resorption ohne Zweifel begünstigt. Nach Entfernung des Pflasters schwanden die Reizungserscheinungen bald wieder. Es braucht kaum bemerkt zu werden und zum Ueberfluss lehren es noch die beiden anderen Fälle, dass diese Erscheinungen mit dem Firnissen an und für sich,

1) Bekanntlich sind es gerade „Rheumatismus"-Kranke, welche sich - zum Hohn für jene Theorie - am liebsten die Haut mit imperspirablen Veberzügen (Gichtpapier ètc.!) bedecken. 
mit der sogenannten „Unterdrückung der Hautfunction“, Nichts zu thun haben.

Im Fall Il war das Einzige, was während der Einwickelung auffel, eine Zunahme der Harnmenge. Leider ist vorher die 24stündige Haramenge nicht gemessen worden, jedoch nur, weil sie nicht auffallend gross erschien. Man darf diese Zunahme wohl als eine wirkliche Folge der Unterdrückung wenn auch nicht der gesammten Hautthätigkeit, so doch der Wasserverdunstung betrachten, die einzige Folge, welche ich beim Menschen gesehen habe, zugleich eine Bestäligung für das schon im Eingang Gesagte, dass nehmlich die Leistungen der Haut als Ausscheidungsorganes mit Leichtigkeit von anderen Organen übernommen werden können. Constant ist übrigens, nach der vorbergehenden Beobachtung zu-schliessen, auch die Urinvermehrung nicht, vielleicht weil die Wasserausgabe durch die Haut schon iberhaupt grossen Schwankungen unterworfen ist, oder weil sich an der Ersatzleistung ausser den Nieren noch andere Organe (Lungen, Darm) betheiligen. Vielleicht endlich hat in Fall I auch der Gebrauch des Morphiums (der Pat. nahm jeden Abend eine ganz geringe Menge davon mit Aq. amygd. am.) die Harnabsonderung beschränkt.

Fall III bedarf kaum eines erläuternden Zusatzes, denn es ist gar keine Erscheinung aufgetreten, welche als besondere Wirkung des Firnissens zu betrachten gewesen wäre. Wir hatten gerade hier in Anbetracht der reizenden Wirkung des Theers, der grossen Ausdehnung und der langen Dauer, in welcher er zur Anwendung kam, erwartet, dass wenigstens anf der Höhe der Firnissung eine Reizung der Harn ausführenden Schleimbäute, ühnlich wie in Fall I eintreten würde. Allein auch nicht einmal diese trat ein, während doch der Urin die bekannte dunkle Färbung und einen Geruch nach Theer annahm. Ich erkläre mir dies daraus, dass von den flüchtigen Bestandtheilen des Theeres, welche die Färbung und den Geruch bedingen (Carbolsäure), ein geringer Theil durch die Athmung aufgenommen wurde, während die Aufsaugung dieser und anderer nicht flüchtiger reizender Stoffe von der Haut aus viellelcht durch die chronische Erkrankung derselben erschwert war. Für die Frage nach der Wirkung des Firnissens ist natürlich gleichgültig, ob die Aufsaugungsthätigkeit der Haut gehemmt war, oder nicht, da hier vielmehr jhr Ausscheidungsvermögen in Betracht kommt. Sollte 
auch diese durch die Krankheit beeinträchtigt gewesen sein, so hätte das Firnissen erst recht müssen schädlich wirken - wenn es eben schädliche Wirkungen beim Menschen hätte.

Was endlieh die Wirkung auf die Körpertemperatur betrifft, die einzige Wirkung, welche ich in meinen früheren an Fiebernden angestellten Versuchen einige Mal beobachtet hatte (s. S. 187), so war in den neuen Versuchen auch diese nicht zu erkennen, und wenn ich schon damals auf die Temperatur herabsetzende Wirkung des "Firnissens" bei Menschen, zumal in therapeutischer Beziehung keinen Werth legte ${ }^{1}$ ), so dürtte ich jetzt vollends mit der Ansicht im Reebt erscheinen, dass jeder ursächliche Zusammenhang zwischen Hautfirnissung und Veränderungen der Körpertemperatur bei Menschen von der Hand zu weisen sei. Unbeschadet dieser Ansicht aber glaube ich jene früher beobachteten Temperatursenkungen nicht als irrthümliche, oder ganz zufällige betrachten zu müssen. Einen Irrthum kann ich schon deshalb ausschliessen, weil ich selbst damals die Messungen vor und nach dem "Firnissen" gemacht habe und ein so einfaches Verfahren, wie das Messen der Achseltemperatur nicht leicht zu Täuschungen Anlass giebt. Ebensowenig kann ich einen blossen Zufall annehmen, wenn, wie es einige Mal vorgekommen ist ${ }^{2}$ ), die Temperatur bald nach dem Firnissen um mehrere Zehntel-Grade $\left(0,3-0,9^{\circ} \mathrm{C}\right.$. $)$ niedriger, als unmittelbar vorher gefunden wird, namentlich wenn dies, wie hier, bei einer Krankheit, dem Abdominaltyphus, gefunden wird, welche durch einen typischen Temperaturverlauf, wie wenige andere Krankheiten, ausgezeichnet ist, wenn es ferner zu einer Tageszeit gefunden wird, zu welcher die Temperatur nicht die Neigung $\mathrm{zu}$ fallen, sondern zu steigen bat und wenn endlich irgend welche andere die Temperatur beeinflussende Umstände ausgeschlossen sind. Ich halte vielmehr jene Temperatursenkungen allerdings als Wirkungen des „Firnissens", aber nur als Nebenwirkungen hervorgerufen durch gewisse bei der von mir geübten Methode des Firnissens mit ihm verbundene, es begleitende, aber nicht nothwendig dazu gehörende Umstände, nehmlich die längere Entblössung des Körpers bei Zimmertemperatur und die Verdunstung des Aethers

1) I. c. S. 203.

${ }^{3)}$ I. c. S. 197 Fall 1. 
(oder Chloroforms bei Anwendung von Traumaticin). Die Wämeentziehung, welche diese beiden Umstände einzeln oder im Verein mit einander ausüben, kann natürlich unter verschiedenen Verbältnissen verschieden gross sein und kann auf die Achseltemperatur einen verscbiedenen oder auch gar keinen erkennbaren Einfluss ausuben, was nicht weiter auseinandergesetzt $z u$ werden braucht. Dass sie in der Weise, wie sie bei meinen Beobachtungen stattfand, bei nichtfiebernden Personen gar keinen, bei fiebernden dagegen einige Mal einen sichtbaren Einfiuss, eine Herabsetzung der Achseltemperatur, hatte, findet seine Erk]ärung in der bekannten alltäglichen Erfahrung, das Fiebernde viel empfindlicher gegen alle auf die Temperatur wirkenden Einflüsse sind, dass ihre Temperatur viel beweglicher ist, als bei Nichtfiebernden.

In dieser Beziehung ist auch der oben mitgetheilte Versuch mit Collodiumpinselung eines Kaninchens sebr lehrreich. Die erste Pinselung nehmlich, welche sich nur auf Rücken- und Seitentheile des Thieres erstreckte, bewirkle ein sofortiges Abfallen der Mastdarmtemperatur um beinahe $2^{\circ} \mathrm{C}$., das im Verlauf eines Tages wieder vollständig ausgeglichen war ohne jede weitere Störung. Der durch das Firnissen gesetzle Eingriff in die Hautthätigkeit war nicht bedeutend genug, um das Thier ernstlich krank za machen, oder gar zu tödten, aber die Verdunstung des Aethers, die bessere Leitung und Strahlung der Wärme von der gefirnissten Hautfläche ${ }^{1}$ ) waren hinreichend, um bei dem kleinen Thiere eine Ablühlung zu bewirken, welche vorher das Scheeren der Haare allein nicht hatte bervorbringen können.

Ein Kaninchen, welchem die Haare geschoren sind, ist eines wichtigen Mittels für die Regulirung semer Körperwärme beraubt und da es seiner Kleinheit wegen ohnehin eine übermässig grosse Oberfläche darbietet, so wird es der Einwirkung der umgebenden Temperatur nur einen ganz geringen Widerstand entgegensetzen können, seine Temperatur wird abnorm beweglich werden, etwa so, wie es bei Fiebernden der Fall ist. Wenn also bei einem solchen Kaninchen durch Pinseln mit Collodium (oder vielleicht schon durch Aether allein) eine beträchtliche Temperaturherabsetzung sich erreichen lässt, so kann wohl etwas Aehnliches auch

1) Vgl. Krieger, Ztschr. f. Biol. v. S. 476. 
bei fiebernden Menschen erwartet werden, zumal bei Kindern, die sich ja, was das Verhältniss ihrer Oberfläche zu ihrem Gewicht betrifft, mehr den Thieren nähern und auch in der That gegen Wärmeentziehungen empfindlicher sind, als Erwachsene. Indessen da uns einfachere und wirksamere Methoden zur Abkühlung zu Gebote stehen, so glaube ich jetzt so wenig wie früher, dass die Collodiumpinselungen als Abkühlungsmittel im Allgemeinen zu empfehlen seien. Nur das wird sich als practische Consequenz aus den vorstehenden Versuchen und Beobachtungen ergeben, dass, wenn man aus irgend welchen Gründen Collodium anwenden will, man eine noch so ausgedebnte Aufpinselung desselben wegen Unterdrückung der Hautthätigkeit nicht $z u$ scheuen hat und dass es in fieberhaften Krankheiten noch ausserdem als wärmeentziehendes Mittel eine mehr erwünschte, als unerwünschte Nebenwirkung hat.

Im Uebrigen lege ich allen vorstehenden Erörterungen über das Firnissen der Haut bei Menschen einen grossen practischen Werth nicht bei, denn die medicinisehe Praxis hat niemals aufgehört, wie jch oben schon ausgefübrt habe, die menschliche Haut zu "firnissen", so oft und so viel es ihr nöthig schien und sie hat sich davon nichl abhalten lassen, ob auch die Theorie von der gefährlichen Unterdrückung der Hautthätigkeit durch das „Firnissen“ bisher unangefochten blieb. 